Proceeding of the $1^{\text {st }}$ ICEENG conference, 24-26 March, 1998.

\title{
OPERATIONAL PROBLEMS IN BUILDING A COMPUTER NETWORK IN AN ARABIZED ENVIRONMENT
}

\author{
M.Samy Gamal El Din* \\ Aly El Moghazy** \\ Wafae Baghdady***
}

\section{ABSTRACT}

This paper is concerned with building wide area computer networks. The environments concerned in this paper are: (1) Heterogeneous computer facilities. (2) Common resources for communications based in both packet switched and circuit switched networks. (3) Different implemented Arabic codes.

The main objective of this paper is to define recommendations concerned with building a wide area computer network in the above mentioned environments.

This paper could be considered as a useful guide to engineers and researchers in the field of wide area computer networks in a typical heterogencous environment.

\section{I - INTRODUCTION}

The term computer network means an interconnected collection of autonomous computers. Two computers are said to be interconnected if they are able to exchange information. Nowadays computer networks are collection of homogenous (same vendor or $100 \%$ compatible) and heterogeneous (different vendors, different models of the same vendor) computers connected together through computer communication subnetworks to make many new applications feasible for all the network users.

The main goals of computer networks could be summarized as follows $\mid 1,2,3$, 11]: (1) Resource sharing which means all programs, data, and other resources should be available to any one of the network without regard to physical location of the resource and the user. (2) Load sharing. (3) High reliability by having alternative resources of computation facilities. (4) A powerful communication medium among widely separated computer users. The main applications of computer networks are (1) Access to remote programs. (2) Access to remote databases. (3) E Iectronic mailing. The other main objectives of connecting computer systems in a network are to provide network users the following services: (1) File to File transfers. (2) Terminal Emulation. (3) Remote Job entry.

Designers and specialists in this field have met several problems that delay the implementation of computer networks in an arabized environment. Such problems are due to heterogeneous computer facilities, the non use of common resources for

- Dr. Eng., Department of Computers, Military Technical College, Cairo, Egypt.

** Dr. Eng.., Information Systems department

***Dr. Eng., Signal Department 
communications based on both packet switched and circuit switched networks, and different implemented Arabic codes.

Section II in this paper is concerned with the actual environments. Computer communication protocols and architectures problems and recommendations are given in section III. Section IV is concerned with handling of computer arabization over computer networks, problems, and recommendations. Section V deals with theinfrastructure communication environments, problems, and recommendations. Conclusion and future work are given in section VI.

\section{II - ACTUAL ENVIRONMENTS}

The actual arabized environments could be summarized in the following:

1 - Isolated computer systems are serving in different locations and sites.

2- Different sites may use the same application or data.

3- The data is transferred between sites manually or on a magnetic media.

4- Computers at different sites, in general, are not fully utilized.

5- Each site uses its own computer network architecture and also its own arabic code. The used network architecture and arabic code depend mainly on the vendor.

6- There is no efficient use of the infrastructure communication networks which are available such as Egyptnet and others. Egyptnet depends mainly on packet switching technology.

7- There is no standard arabic code to be used by all computer vendors. Each vendor develops his own arabic code to be running on his computer system and its facilities.

8- The Open System Interconnection (OSI) standardized by the International Standarads Organization (ISO; as a reference model is not yet implemented or even defined totally by any of computer vendors. However the so called Transfer Control Protocol/Internetwork Protocol (TCP/IP) product is a de facto standard for many vendors. This product is the equivalent of layer three and layer four in the ISO-OSI model.

9- The infrastructure communication networks are not fully utilized.

\section{III- COMPUTER COMMUNICATION PROTOCOLS AND ARCHITECTURES}

\subsection{Environments}

The OSI model is introduced as a model architecture and a frame work for standards. In this model, the communication functions are described and implemented by viewing the communication task in terms of a column of layers. Each of which contains protocols. Although the OSI model is almost universally as the frame work for discourse in this area. there is another point of view which grows out of the extensive research and practical experience of ARPANET. This view point, which is characterized by a hierachy of protocols, is also presented. Besides OSI model and ARPANET architectures, two commercial architectures are presented. They are IBM System Network 
Architecture (SNA) and DEC Digital Network Architecture (DNA). Details of the standards being developed by various organizations involved by various organization are described in $[4,5,7]$.

The reference model of the OSI as the ISO calles it, has seven layer. They are physical layer, data link layer, network layer, transport layer, session layer, presntation layer, and application layer. Details of the layers functions could be found in $[3,4,11]$.

The Department Of Defence (DOD) has developed its own architecture. Both OSI and DOD protocol architecture (DPA) deal with communications among hetrogeneous computers. Both are based on the concept of protocol and have many similarities. However, there are philosophical and practical differences between the OSI model and the DPA [4, 5, 8, 12].

The SNA consists of five layers. They are data link control, path control, transmission control, data flow control, and function management data services. The physical layer is not addressed directly in SNA and assumed existing in data link layer. The protocol specified for the data link layer is SDLC. For more details see $[7,10,12]$.

DNA appears to have been heavily influenced by ARPANET. Consequently, DNA is closer in spirit to DPA than to the OSI model. DNA is composed of four layers. They are data link layer, transport layer, network services protocol layer, and network application layer.

\subsection{Problems}

1. The existance of different communication architectures and protocols depending on the vendor.

2. The non completness of the definition of the ISO-OSI model which leads to the lack of implementable reference model.

\subsection{Recommendations}

1. The Problems due to the existence of heterogeneous computer communication architecture over a network could be overcome by the following:

A-Follow up of implementation statues of OSI-ISO model by

Different computer vendors.

B- Use of TCP/IP as intermediate de facto standard.

2. Moreover an experimentation phase should be planned and performed by expertise in this area. The objective is to gain a practical view concerning problems of interoperability and how to overcome them considering the actual enviromment and its constraints.

\section{IV- HANDLING OF COMPUTER ARABIZATION OVER COMPUTER NETWORKS}

\subsection{Environments}

1. Generally, the computer arabization should meet the following features:

a- Arabic support for ready made packages and applications software programs. 
b- Provision of bilingualism, i.e. the arabization should provide contextual analysis (to give the correct shape of each character). Latin and arabic screen directions (left to right and right to left), insertion of one language into the otherm natural entry of arabic numbers, split screen, full set of diacritics, extended interrupts and user definable functions and character sets.

c- Provision of transparency, i.e. arabization should allow the use of latin only software to process latin, arabic, and bilingual data, instantly and without any modifications to the software.

d- Realization of portability i.e. arabization can run on different hardware at different environments and provides the same services.

e- Character set should cope to one of the different arabic standards such as ARCII, ASM, SASO, and CODAR-UFD. Examples of these codes are given in [13].

f- Keep Compatibility with different peripherals (screens and printers) from different vendors.

g- Not affecting processing speed of latin applications only.

2. The extension of realizing the above mentioned features is different from one vendor to another. Generally, computer vendors are implementing the arabization at the lower levels of their hardware and software components.

\subsection{Problems and Recommendations}

1- Although the communication services are easily realized for both special and general purpose homogeneous systes, conversions and other similar efforts rnust be devoted towards realizing these services for hetrogenous system.s. As an example, terminal emulation and remote jobs entry services allow terminals connected to a computer to be connected to ano'ther different computer through the network. In heterogeneous systems erivironment, terminals required to $\log$ into different computers with. different characteristics might have to run different terminal emulation (conversion) programs. The loading of these programs on the terminal data and storage memory limit the processing speed and available storage of the terminal.

2- In addition, computer arabization makes restrictions of handling arabic data over the network due to the fact that implementation of arabization such as number of bits for arabic characters, arabic standard hardware and/or software levels is different from vendor to vendor.

3- The use of different arabic codes over a network could be overcome by the following:

a- Use a "standard" arabic code to be agreed upon over the infrastructure computer communication networks.

b- The vendors should supply users with an arabic code program conversion to and from the code used in the infrastructure communication subnetworks. 
Proceeding of the $1^{\text {st }}$ ICEENG conference, $24-26$ March, 1998 .

CE. 3.339

\section{V - COMMUNICATION SUBNETWORK}

\subsection{Environments}

The communication subnetwork consists of nodal switching and transmission supports. The switching nodes are bsed on either circuit switching technology or packet switching technology. The users, computers and terminals, access the packet switching nodes through the circuit switching network (either in direct mode or in dial-up mode). The packet switching nodes are intereonnected through the eircuit switching network in direct mode. The relevant CCITT recommendations applicable to environments are as follows:

1- Access of users to packet switching nodes:

a- CCITT X25 for access of packet mode Data Terminal Equipment (DTEP) in direct mode. The CCITT X25 defines various physical levels among them V24/V28 and V35. The data link layer is based on ISOOSI High Level Data Link Control (HDLC) and called LAP-B.

b- CCITT X32 for access of DTE-P through dial up lines. The data link layer is LAP-X.

c- CCITT X23, X29 for access of asynchronous DTE to the packet switching nodes. The part of packet switching node which allows this access is called Packet Assembly Disassembly (PAD). CCITT X3 - defines about 20 parameters that accomdates different DTE characteristics.

2- Interconnection of packet switching nodes: The ('C Tl"l does not delinc a protocol for such interconnection since it is transparent to the network users.

3- If the user DTE is colocated with the packet switching nodes, moderns (or equivalent device if the circuuit switching network is based on $64 \mathrm{kbps}$ digital channel) have to be used.

The modern characteristics has to be defined at the level of network administration. The services offered to the users of packet switching network are defined in CCITT X2. These services are reflected in the user subscription form.

\subsection{Problems}

1. It is worthy to emphasize that, although the packet switching network offers reliable data transmission services to their users, it does not completely solve the problem of interoperability between hetrogenous DTS's. The interoperability is assured at higher level protocols.

2. Even CCITT issues recommendation for access to packet switching network each 4 years, the implementations of these recommendations by the vendors of both network components and DTE's are not unique.

3. Management and security issues in public packet switching network are hidden from network users. 


\subsection{Recommendations} recommends:

To overcome the "problems" stated in section 5.2 here above, one

1- The users of packet switching networks must be aware that such network does not allow hetrogenous hosts to transfer information between them. This network however allows finite level of interconnectivity that is not provided by circuit switching technology, namely:

a- Connection of asynchronous DIE to synchronous DTE with different speeds.

b- Simultaneous connection (through virtual circuits) of one synchronous DTE to several synchronous DTE with different speeds.

c- Using the PAD function, different synchronous terminal characteristics could be acommandated to a specific host by choosing the proper PAD profile defined in CCITT X3.

2- In acquistion phase of DTE's one should define and test the profile of access to the packet switching networ'k.

3- If security is a main issue for a certain user, he should implement the security mechanism "in his site" either using additive software or equipements.

\section{VI - CONCLUSION AND FUTURE WORK}

The main goal of this paper is to recommend an applicable solutions for problems which are facing the interoperability of computer networking in an arabized domain. The recommendations are concerning the architectural, communication network and arabic codes aspects. These recommendations could serve as a guide in achieving interoperability in the above mentioned environments. Finally we recommend an experimentation phase to be started immediatly to apply the cited recommendations.

\section{REFERENCES}

[1] Amer Fathy and Yao-Nan Lien. "Design and Performance Evaluation of A Hierarchical Hybrid Adapative Routing Algorithm for Large Scale Computer Communication Networks. "Tech. Report OSU-CISRC-9/87-TR27, Dept. of Comp. and Info. Sci. Ohio State Univ.. June 1988.

[2] Amer Fathy and Yao-Nan Lien. "A Comparative Survey of Routing Algorithms for Computer Commiunication Networks" Tech. Report OSU-CISRC-9/87TR27, Dept. of Comr., and Info. Sci., Ohio State Univ.. Sep. 1987.

[3] William Stallings. Data and Computer Communications, second liddition, Macmillan Publishing Company, New York, 1988.

[4] Enni Ennis, G.; Kaufman, D.; and Biba, K. "DOD Protocol Reference Model", Sytek, TR-8202.6, Sept. 1982.

[5] ISO International Organization for Standardization. "Basic Reference Model for Open Systerns Interconnection", DIS 7498, 1983.

[6] John Johnson, S. "Architectural Evolution" Digital Unveils Its Decnet Phase III "Data Communications, March 1980. 
Proceeding of the $1^{\text {st }}$ ICEENG conference, 24-26 March, 1998.

[7] IBM Corp., "Systems Network Architecture: Concepts and Products", IBM GC30-3072, 1981.

[8] Leiner Barry, John Postel, Robert Cole, and David Mills, "The DARA Internet Protocol Suite, "IEEE INFOCOM Proc., PP. 28-36, March 1985.

[9] Quarterman J.S. and J.C. Hoskins, "Notable Computer Networks", ACM Comm., vol. 29, No. 10, PP. 932-971. Oct. 1986.

[10] Schwartz Mischa, "Telecommunication Networks: Protocols, Modeling and Analysis". Addison-Wesley Publishing Co., 1987.

[11] Tanenbaum A.S., "Computer Networks", Prentice-Hall Inc., 1981.

[12] Walker S., "Department of Defence Data Network". Signal, Oct. 1982.

[13] Bachir Halimi, "Transparent Arabization Design, Standards, and Usrs", Proc. of the Fifth Gulf Computer Conference, Dubai, UAE, 1985. 
